\title{
Toward controlling dielectrophoresis
}

\author{
Dong Eui Chang ${ }^{* \dagger} \uparrow$ and Nicolas Petit \\ Centre Automatique et Systèmes, Ecole Nationale Superieure des Mines de Paris 60. \\ bd Saint-Michel, 75272 Paris, Cedex 06, France
}

\section{SUMMARY}

Dielectrophoresis is the motion of a particle due to the interaction between a non-uniform electric field and its induced dipole moment in the particle. With the advent of the fabrication technology at micro/nanoscale, dielectrophoresis is actively applied in manipulating, separating, and characterizing micro/nano-sized particles such as DNA, cells, proteins, nanotubes and nanoparticles. In this paper we introduce control engineers to dielectrophoresis by suggesting several possible research topics and performing a case study: a time-optimal control of a dielectrophoretic system with a state constraint. Copyright (C) 2005 John Wiley \& Sons, Ltd.

KEY WORDS: dielectrophoresis; nanotechnology; biotechnology; systems theory; time-optimal control

\section{INTRODUCTION}

Dielectrophoresis (DEP) refers to the motion of a particle due to the force exerted on the induced dipole moment of the particle by a non-uniform electric field. The study of dielectrophoresis and its application to the manipulation of small and biological particles was first thoroughly investigated out by Pohl [1]. At that time there was a limit to the magnitude of electric fields that could be generated with small voltages. With the advent of MEMS and nanotechnology, one can now generate a large electric field with weak voltages so that dielectrophoresis may be actively applied to manipulating, separating, and characterizing micro/ nano-sized particles such as cells, DNA, proteins, nanotubes, and nanoparticles [2-7]. An advantage of dielectrophoresis over electrophoresis is that it can also work on neutrally charged particles [8].

The objective of this paper is to turn the attention of control engineers to this area of dielectrophoresis so that they can not only find many interesting control problems but also contribute to DEP-based engineering applications. The connection between control theory and dielectrophoresis is not new. The interpretation of a simple model of the induced dipole moment due to an electric field as a control system was briefly mentioned by Daniel [9] in 1967. To our

\footnotetext{
${ }^{*}$ Correspondence to: D. E. Chang, Centre Automatique et Systèmes, Ecole Nationale Superieure des Mines de Paris 60, bd Saint-Michel, 75272 Paris, Cedex 06, France.

†E-mail: dchang@cas.ensmp.fr
} 
knowledge, the first attempt to apply control theory to a DEP problem was made by Kaler $e t$ al. $[10,11]$ for the purpose of locally stabilizing levitation of biological particle with dielectrophoresis. Their main approach was as follows. They linearized the original nonlinear dynamics around an equilibrium of interest, applied a sinusoidal voltage on electrodes at an appropriate frequency, (naively) averaged the resultant equations over the period of the sinusoidal voltage so that the equations become time-invariant, and then finally modulate the amplitude of the boundary voltage, which was initially assumed constant, with a linear feedback controller. Their clever but ad hoc procedure proved effective experimentally [10,11]. Since their work has been unknown to much of the control community, the approach has neither been formalized nor improved by modern control theory. Only recently, the issue of applying control technology to DEP applications was raised in Reference [12]. Hence, it is time for the control theory, which has advanced for the last 40 years, to make contributions to this area. For an overview of various control issues in other (non-DEP-related) nano-scale systems, we refer to the report available on the web page [13].

This paper is organized as follows. First, we explain the physics of dielectrophoresis and review a traditional method of manipulating particles with dielectrophoresis in Section 2. Second, we provide several possible research topics for control engineers: the system identification, the boundary control of DEP systems governed by PDEs, effect of a term quadratic in a control variable, and higher-order hidden dynamics. Third, we perform a case study: a time-optimal control of a DEP system with a state constraint that arises from the existence of electrodes. According to Chang et al. [14], all the time-optimal trajectories in the system without the state constraint begin with undershoots. Hence, one needs to do the timeoptimal study with the state constraint to prevent particles near the electrodes from trying to go through electrodes. Finally, we conclude in Section 5.

\section{BACKGROUND MATERIALS}

\subsection{Physics of dielectrophoresis}

We briefly explain basic physics of dielectrophoresis; see References $[1,8,15]$ for more details. When a particle is immersed in a medium and an electric field, $\mathbf{E}(\mathbf{x}, t)$, is imposed, then an effective dipole moment, $\mathbf{m}(\mathbf{x}, t)$, is induced in the particle, where $\mathbf{x} \in \mathbb{R}^{3}$ is the position vector and $t$ is the time; see Figure 1(a). The relation between $\mathbf{E}$ and $\mathbf{m}$ is linear and given by

$$
\mathbf{m}(\mathbf{x}, t)=g(t) * \mathbf{E}(\mathbf{x}, t)
$$

where * denotes time convolution. The Laplace transform $G(s)$ of $g(t)$ is called the ClausiusMossotti function (up to a constant) $[1,8,15]$ and it depends on the physical structure and electric properties of the particle and the electric properties of the medium in which the particle is immersed. When the particle is a sphere, $G(s)$ is rational, generically of relative degree 0 , where the degree of the denominator (or numerator) is the number of layers in the particle; see Figure 1(b) and Appendix $\mathrm{C}$ of Reference [8]. For example, when the particle is spherical and homogeneous, $G(s)$ is given by

$$
G(s)=a+\frac{b}{s+c}
$$




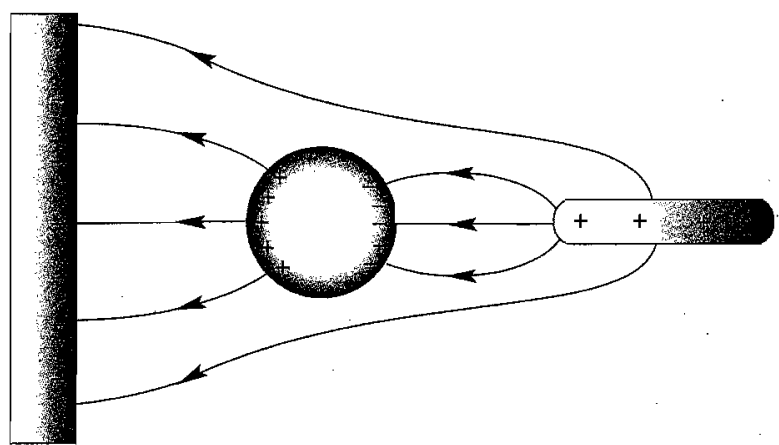

(a)

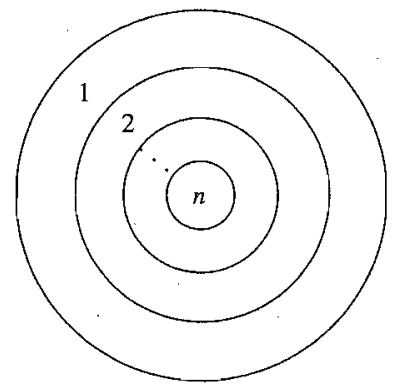

(b)

Figure 1. (a) An electric field redistributes the charges in the particle so that a dipole moment is induced in the particle; and (b) a multi-layer shell model of a spherical dielectric particle. The Clausius-Mossotti (transfer) function of the $n$-layered spherical particle in a medium is a rational function of relative degree 0 where the degree of the denominator is $n$.

with

$$
\begin{gathered}
a=4 \pi r^{3} \varepsilon_{m} \frac{\varepsilon_{p}-\varepsilon_{m}}{\varepsilon_{p}+2 \varepsilon_{m}} \\
b=a\left(\frac{\sigma_{p}-\sigma_{m}}{\varepsilon_{p}-\varepsilon_{m}}-\frac{\sigma_{p}+2 \sigma_{m}}{\varepsilon_{p}+2 \varepsilon_{m}}\right) \\
c=\frac{\sigma_{p}+2 \sigma_{m}}{\varepsilon_{p}+2 \varepsilon_{m}}
\end{gathered}
$$

where $r$ is the radius of the particle, $\varepsilon_{p}$ (resp., $\varepsilon_{m}$ ) is the permittivity of the particle (resp., medium) and $\sigma_{p}$ (resp., $\sigma_{m}$ ) is the conductivity of the particle (resp., medium). The frequency dependence of the Clausius-Mossotti function $G(s)$ is at the heart of DEP applications since most methods of separating particles with DEP make use of the fact that different types of particles have different frequency dependences $[4,5,8]$.

The dielectrophoretic force, $\mathbf{F}_{\mathrm{dep}}$, due to the interaction between the induced dipole moment $\mathbf{m}$ and the electric field $\mathbf{E}$ is given by

$$
\mathbf{F}_{\mathrm{dep}}(\mathbf{x}, t)=(\mathbf{m}(\mathbf{x}, t) \cdot \nabla) \mathbf{E}(\mathbf{x}, t)
$$

The dielectrophoretic torque, $\tau_{\text {dep }}$, is given by

$$
\tau_{\text {dep }}(\mathbf{x}, t)=\mathbf{m}(\mathbf{x}, t) \times \mathbf{E}(\mathbf{x}, t)
$$

In applications of dielectrophoresis there are electrodes that govern the boundary voltage, which induces the electric field $\mathbf{E}(\mathbf{x}, t)$, so the boundary voltage plays the role of control. 


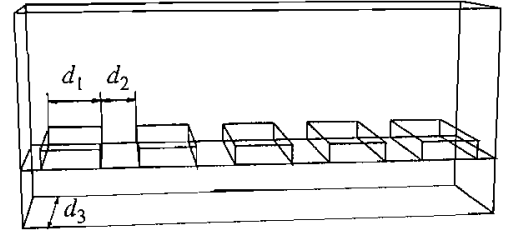

(a)

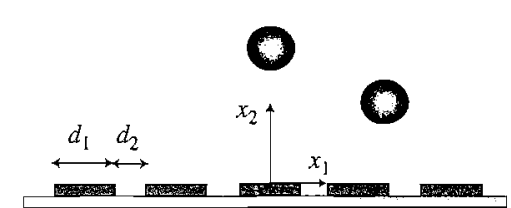

(b)

Figure 2. (a) The arrangement of a linear electrode array; and (b) the front view of the arrangement of a linear electrode array where the origin of the $x_{1} x_{2}$ axis lies in the middle of an electrode.

\subsection{Parallel array of linear electrodes}

We consider the configuration with a parallel array of linear electrodes in Figure 2(a). This configuration is often used to separate one type of bioparticles from the rest in the mixture of particles, or to sort bioparticles $[3,5,16]$. As particles are usually relatively small compared with the electrodes, one may assume that each electrode is infinitely long (or, $d_{3} \gg d_{1}, d_{2}$ ) and that there are infinite number of them. Then, the problem reduces to a planar case as in Figure 2(b). On electrodes we give the following boundary value of the potential function (or, the voltage):

$$
V_{b \mathrm{~d}}\left(x_{1}, t\right)=u_{l}(t), \quad x_{1} \in\left[l\left(d_{1}+d_{2}\right)-d_{1} / 2, l\left(d_{1}+d_{2}\right)+d_{1} / 2\right]
$$

with $u_{l}(t) \in \mathbb{R}, l \in \mathbb{Z}$. We choose $u_{l}(t)$ 's such that the function $V_{\text {bd }}$ is even and periodic in $x$ of period $N\left(d_{1}+d_{2}\right)$ with a fixed $N \in \mathbb{N}$, i.e.

$$
V_{\mathrm{bd}}\left(x_{1}, t\right)=V_{\mathrm{bd}}\left(-x_{1}, t\right)=V_{\mathrm{bd}}\left(x_{1}+N\left(d_{1}+d_{2}\right), t\right)
$$

It is practical to assume that the boundary value of the potential function between electrodes changes linearly as follows:

$$
\begin{gathered}
V_{\mathrm{bd}}\left(x_{1}, t\right)=\frac{u_{l+1}(t)-u_{l}(t)}{d_{2}}\left(x_{1}-l\left(d_{1}+d_{2}\right)-\frac{d_{1}}{2}\right)+u_{l}(t) \\
x_{1} \in\left[l\left(d_{1}+d_{2}\right)+d_{1} / 2,(l+1)\left(d_{1}+d_{2}\right)-d_{1} / 2\right]
\end{gathered}
$$

with $l \in \mathbb{Z}$. This assumption is acceptable when the gap between electrodes is small (see 10.3 .2 of Reference [5] and references therein).

The potential function $V\left(x_{1}, x_{2}, t\right)$ in the region $x_{2}>0$ is derived by solving the Dirichlet problem, $\nabla^{2} V=0$ with the boundary condition given above. As the boundary value of the voltage is a linear combination of $u_{l}$ 's, the potential function $V\left(x_{1}, x_{2}, t\right)$ is also a linear combination of $u_{l}$ 's. It can be written as follows:

$$
V\left(x_{1}, x_{2}, t\right)=\sum_{l=1}^{N} u_{l}(t) V_{l}\left(x_{1}, x_{2}\right)
$$

Hence, the electric field $\mathbf{E}\left(x_{1}, x_{2}, t\right)$ is also a linear combination of $u_{l}(t)$ :

$$
\mathbf{E}\left(x_{1}, x_{2}, t\right)=\sum_{l=1}^{N} u_{l}(t) \mathbf{E}_{l}\left(x_{1}, x_{2}\right)
$$


with $\mathbf{E}_{l}=-\nabla V_{l}$. By (1)-(6), the induced dipole moment, the dielectrophoretic force and the dielectrophoretic torque on a particle with the Clausius-Mossotti function $G(s)$, are, respectively, given by

$$
\begin{gathered}
\mathbf{m}\left(x_{l}, x_{2}, t\right)=\sum_{l=1}^{N}\left(g * u_{l}\right)(t) \mathbf{E}_{l}\left(x_{1}, x_{2}\right) \\
\mathbf{F}_{\text {dep }}\left(x_{l}, x_{2}, t\right)=\sum_{l=1}^{N} \sum_{m=1}^{N}\left(g * u_{l}\right)(t) u_{m}(t)\left(\mathbf{E}_{l}\left(x_{1}, x_{2}\right) \cdot \nabla\right) \mathbf{E}_{m}\left(x_{1}, x_{2}\right) \\
\tau_{\text {dep }}\left(x_{1}, x_{2}, t\right)=\sum_{l=1}^{N} \sum_{m=1}^{N}\left(g * u_{l}\right)(t) u_{m}(t) \mathbf{E}_{l}\left(x_{1}, x_{2}\right) \times \mathbf{E}_{m}\left(x_{1}, x_{2}\right)
\end{gathered}
$$

where $g(t)$ is the inverse Laplace transform of the Clausius--Mossotti function $G(s)$.

\subsection{Traditional methods of manipulating particles}

In the current application area of dielectrophoresis, sinusoidal signals are often used for the boundary potential to manipulate/separate particles $[16,17]$. Sinusoidal signals have a couple of advantages in that they are not only easy to generate but also make use of the linear relation between the induced dipole moment and the electric field in (1).

We consider the case of controlling particles with a travelling wave array from References $[17,18]$. Notice the four-phase travelling wave electrode array in Figure 2 with the boundary potentials, $\phi_{1}\left(x_{1}, 0\right)$ and $\phi_{2}\left(x_{1}, 0\right)$ in Figure 3, where we assume that the potentials change linearly between neighbouring electrodes on the boundary $[5,17]$. The potential on the boundary is time-modulated as follows:

$$
\phi\left(x_{1}, 0, t\right)=\phi_{1}\left(x_{1}, 0\right) \cos (\omega t)+\phi_{2}\left(x_{1}, 0\right) \sin (\omega t)
$$

One computes the corresponding dielectrophoretic force by (5) or (8), and then takes the naive average of it over the period $2 \pi / \omega$ (when one wants to justify the use of the averaging method,

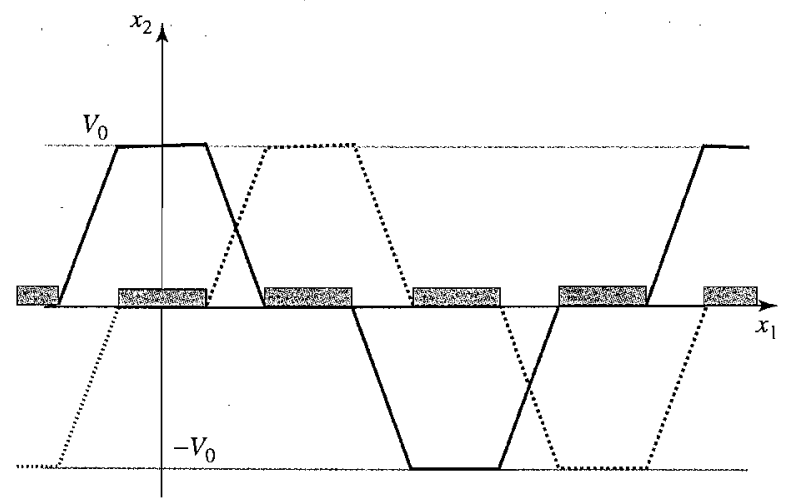

Figure 3. The boundary condition for the potential at $x_{2}=0$ for the travelling wave electric field. The potential $\phi_{2}\left(x_{1}, 0\right)(\cdots)$ is a one-phase shift of the potential, $\phi_{1}\left(x_{1}, 0\right)(-)$. 


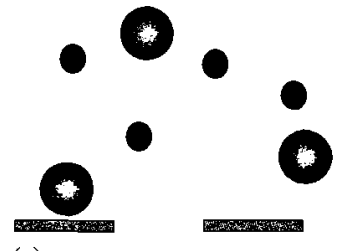

(a)

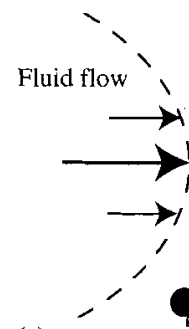

(c)

Figure 4. Separation of particles with dielectrophoresis and field flow fractionation: (a) a mixture of two different types of particles before the DEP force is given; (b) the vertical separation is achieved with the DEP force; and (c) fluid flow collects one type of particles while the other type stays attracted to the electrodes.

the dynamics needs to be transformed to a standard form [19], but this procedure is missing in this traditional approach). The averaged dielectrophoretic force $\left\langle\mathbf{F}_{\text {dep }}\right\rangle$ is of the form

$$
\left\langle\mathbf{F}_{\text {dep }}\right\rangle\left(x_{1}, x_{2}\right)=\operatorname{Re}[G(\mathrm{j} \omega)] F_{c}\left(x_{1}, x_{2}\right)+\operatorname{Im}[G(\mathrm{j} \omega)] F_{s}\left(x_{1}, x_{2}\right)
$$

which can be checked in References [17,18] for more details. In general, the term $\operatorname{Re}[G(\mathrm{j} \omega)] F_{c}\left(x_{1}, x_{2}\right)$ in $(10)$ creates a vertical force and the term $\operatorname{Im}[G(\mathrm{j} \omega)] F_{s}\left(x_{1}, x_{2}\right)$ creates a horizontal force $[4,19]$. Consider a mixture of two different types of particles immersed in a fluid medium in Figure 2. Each type will have different Clausius-Mossotti functions $G(s)$. By choosing an appropriate frequency $\omega$, one can separate these two kinds of particles. One can also employ additional fluid flow to move particles horizontally instead of using the term $\operatorname{Im}[G(\mathrm{j} \omega)] F_{s}\left(x_{1}, x_{2}\right)$. This method with fluid flow is called the field flow fractionation [4, 5]; see Figure 4. This traditional method works well experimentally. Its formalization and improvement are left for control engineers.

\section{RESEARCH DIRECTIONS FOR CONTROL ENGINEERS}

We now propose several possible research topics for control engineers in the field of dielectrophoretic systems. This section is inspired by Jones [8], a standard reference in dielectrophoresis.

First, we consider a simple system which has all the key features of dielectrophoretic systems. The configuration is given in Figure 2 with the boundary voltage as in Figure 5. Notice that we here consider the exact (not approximate) boundary condition, $\partial \phi / \partial n=0$ between electrodes. 


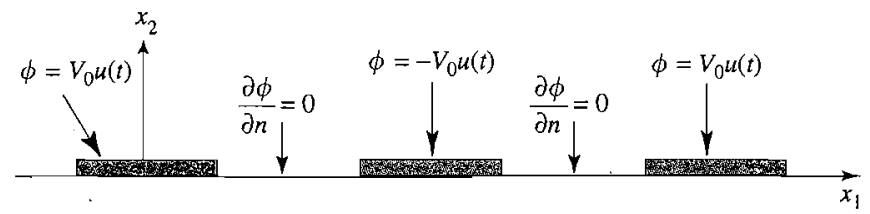

Figure 5. The exact boundary condition for the potential at $y=0$ for the standing wave electric field. The normal derivative of the potential is zero on the boundary between neighbouring electrodes.

We impose the boundary potential $V_{0} u(t)$ on every other electrode and $\left(-V_{0} u(t)\right)$ on the others where $u(t)$ is the control. There is a neutrally charged spherical particle in a fluid medium in the chamber. We assume that the particle is homogenous such that the Clausius-Mossotti functions $G(s)$ is given by (2) with $a \neq 0$. The dielectrophoretic force $\mathbf{F}_{\text {dep }}(x, y, t)$ is of the form:

$$
\mathbf{F}_{\text {dep }}\left(x_{1}, x_{2}, t\right)=u(t)(g * u)(t)\left(\mathbf{E}\left(x_{1}, x_{2}\right) \cdot \nabla\right) \mathbf{E}\left(x_{1}, x_{2}\right)
$$

where $u(t) \mathbf{E}\left(x_{1}, x_{2}\right)$ is the electric field in $x_{2}>0$; see Reference [18] to verify this. Function $g(t)$ in (11) is the inverse Laplace transform of $G(s)$ in (2) and given by

$$
g(t)=a \delta(t)+b \mathrm{e}^{-c t}
$$

where $\delta(t)$ is the Dirac delta function. Thus, the DEP force in (11) can be written as

$$
\mathbf{F}_{\text {dep }}\left(x_{1}, x_{2}, t\right)=\left(a u(t)^{2}+b y(t) u(t)\right)\left(\mathbf{E}\left(x_{1}, x_{2}\right) \cdot \nabla\right) \mathbf{E}\left(x_{1}, x_{2}\right)
$$

where the new variable $y$ satisfies

$$
\dot{y}+c y=u
$$

To simplify the dynamics, we will make two assumptions. First, we assume that the particle and its surrounding fluid are such that the Reynolds number is low. Under this assumption the drag force on a sphere is linear in velocity by the Stokes law; see Section 3.8.1 of Reference [5]. Second, we assume that the term $m \ddot{x}$ is relatively small compared with other forces, which is reasonable as the particle is small and light. For large or heavy particles this assumption fails and the dynamics cannot be simplified. The resulting study requires further investigations which fall out of the scope of this paper. We refer to References [5,20] to help understand these two assumptions. Then, the only remaining forces acting on the particle are the drag, the gravitational plus buoyant force and the dielectrophoretic force. The equation of motion is of the form

$$
\underbrace{m\left[\begin{array}{c}
\ddot{x}_{1} \\
\dot{x}_{2}
\end{array}\right]}_{\text {inert. }=0}+\underbrace{k\left[\begin{array}{c}
\dot{x}_{1} \\
\dot{x}_{2}
\end{array}\right]}_{\text {drag }}+\underbrace{w\left[\begin{array}{c}
0 \\
x_{2}
\end{array}\right]}_{\text {grav.+buoy. }}+\underbrace{\left(a u^{2}+b y u\right)\left[\begin{array}{l}
H_{1}\left(x_{1}, x_{2}\right) \\
H_{2}\left(x_{1}, x_{2}\right)
\end{array}\right]}_{\text {DEP }}=0
$$

with $(\mathbf{E} \cdot \nabla) \mathbf{E}=\left(H_{1}, H_{2}\right)$. Let

$$
\mathbf{x}=\left[x_{1}, x_{2}, y\right]^{\mathrm{T}}
$$


Then, the dynamics can be written in the form familiar to control engineers as follows:

$$
\frac{\mathrm{d}}{\mathrm{d} t} \mathbf{x}=X_{0}(\mathbf{x})+u X_{1}(\mathbf{x})+u^{2} X_{2}(\mathbf{x})
$$

We now discuss features of the dielectrophoretic systems and suggest possible future research directions to control engineers.

1. Quadratic in control. Notice that the system in (14) is not an affine control system. There exists a term which is quadratic in control $u$. In general, the term quadratic in control comes from the fact that the Clausius-Mossotti function $G(s)$ is a rational function of relative degree 0 . The existence of this quadratic term makes dielectrophoretic systems challenging from a control point of view because it does not allow both plus and minus signs of the term.

2. Bounded control. The control $u$ is always bounded in its magnitude because it is a voltage-divided by $V_{0}$, precisely speaking-on electrodes.

3. Boundary control. One can also view DEP systems from the viewpoint of the partial differential equations (PDEs). The PDE involved here is the Laplace equation in computing the potential function from the boundary value where the boundary value is regarded as control. When there are large number of particles, one can also employ a density function to describe their overall movement. For example, the Fokker-Planck equation is used with a periodic potential to separate particles in Reference [21]; see also Section 8.4 of Reference [5] and references therein. Hence, PDE control theory will be useful for this direction of research.

4. System identification. Different types of biological cells or small particles have different physical/electrical characteristics such as the number of layers in the shell model in Figure 1(b), the permittivity, and the conductivity of the particle. Namely, one needs to model the Clausius-Mossotti function $G(s)$; see Appendix C of Reference [8]. Existing measurement techniques of $G(s)$ in application have not fully taken advantage of system identification theory. In Appendix E of Reference [8], one can see that there have been some approximate methods in identifying $G(s)$. For example, they assume that all the poles of $G(s)$ are simple and sufficiently distant from one another so that they, can reduce the system identification problem to the case of a single pole. In addition, the Argand diagram used in Appendix E of Reference [8] is, in principle, the same as the Nyquist plot in control theory. One can see that the system identification technology in control theory will contribute a lot to the study of nanoparticles and bioparticles [7, 10,11]. In particular, the work in References [10,11] is noteworthy because the concept of feedback control through the boundary value was employed to identify the Clausius-Mossotti function of a given bioparticle. In the same references, one can learn that the dielectrophoretic levitation of particles is closely linked with system identification problems in the sense that one needs to levitate and trap a particle to measure its electrical/physical properties; see References [8, Section 3.4, 22] and references therein.

5. Interaction among particles or between particles and electrodes. When particles are close to each other, sometimes the interaction between them is no longer negligible. This interaction creates clustering or chaining of particles; see Chapters 6, 7 of Reference [8]. Likewise, when a particle is close to an electrode, a new chemical force starts to appear due to the interaction between the charges on the electrode and the induced dipole moment in 
the particle. In these cases, one needs to modify the dynamics taking into account these interaction effects; see Chapter 3 of Reference [5], in particular, Section 3.4.

6. Multipoles. Non-uniform electric fields induce not only dipole moments but also multipole moments in a particle; see Chapter 4 of Reference [15] or Appendix B of Reference [8]. One can add this into the dynamics for a more precise model or employ a robust control technique, regarding this higher-order effect as uncertainty.

This list is far from exhaustive. We believe that control technology, which has advanced for the last forty years, will make many contributions to the applications of dielectrophoresis.

\section{A CASE STUDY: A TIME-OPTIMAL CONTROL PROBLEM}

We now consider a time-optimal control problem of a dielectrophoretic system because timeoptimal control is one of the useful and challenging optimal control problems. Ideally, timeoptimal control will reduce the process time in manipulating particles in labs-on-a-chip systems. As an initial step, we will deal with a simple case of (14). For this case the time-optimal control problem was studied in Reference [14] without the state constraint which comes from the fact that particles cannot go through electrodes. In Reference [14], it was discovered that due to the existence of the quadratic term $u^{2}$ in (14), optimal trajectories without the state constraint always start with an undershoot. Because of this phenomenon, it is necessary to consider the state constraint because the particle starting close to electrodes and following the time-optimal trajectory, which is derived without the state constraint considered, will violate the state constraint. We hope that this case study will provide a good example of exchanging problems and solutions between control theory and engineering application.

\subsection{Derivation of equations of motion}

We derive the dynamics for which we will investigate the time-optimal control. First, recall the equation of motion in (12) and (13). From Reference [18] one can check that $H_{1}\left(0, x_{2}\right)=0$ in (13). Hence, the $x_{2}$-axis is an invariant set of the dynamics. As the vertical motion of particles in the whole chamber can be practically represented by that of particles on the $x_{2}$-axis, we will restrict ourselves to this invariant line. Let us assume that the particle is neutrally buoyant, so that the coefficient $w=0$ in (13). Then, the dynamics of $\left(x_{2}, y\right)$ on the $x_{2}$-axis can be written as

$$
\begin{gathered}
k \dot{x}_{2}+\left(b y u+a u^{2}\right) H_{2}\left(0, x_{2}\right)=0 \\
\dot{y}+c y=u
\end{gathered}
$$

where one can verify that $H_{2}\left(0, x_{2}\right)$ satisfies $H_{2}\left(0, x_{2}\right)<0$ for $x_{2}>0, H_{2}(0,0)=0$ and $\lim _{x_{2} \rightarrow \infty} H_{2}\left(0, x_{2}\right)=0$; see Reference [18] to verify this. Let

$$
x=\int_{\epsilon}^{x_{2}} \frac{-k}{b H_{2}\left(0, x_{2}\right)} \mathrm{d} x_{2}
$$

for $x_{2} \geqslant \varepsilon$ where $\varepsilon$ is a positive number. If a particle is close to the electrode, then additional forces other than the DEP force start to appear in the dynamics (for example the Stern layer effect; see Section 3.4 of Reference [5]), so the parameter $\varepsilon$ in (17) defines the region $\left\{x_{2} \geqslant \varepsilon\right\}$ 
where the dynamics (15) is valid. As a function of $x_{2}, x\left(x_{2}\right)$ is strictly monotone on $\left\{x_{2} \geqslant \varepsilon\right\}$ since $x^{\prime}\left(x_{2}\right)=-k /\left(b H_{2}\left(0, x_{2}\right)\right)$ is sign definite on $\left\{x_{2} \geqslant \varepsilon\right\}$. Hence, we can use $x$ as a new co-ordinate in place of $x_{2}$. This new co-ordinate not only simplifies the dynamics but also makes the dynamics independent of the physical size of electrodes (such as $d_{1}$ and $d_{2}$ in Figure 2) and the maximum value of the boundary voltage, $V_{0}$. In the state $(x, y)$, the equations in (15) and (16) are written as

$$
\begin{aligned}
& \dot{x}=y u+\alpha u^{2} \\
& \dot{y}=-c y+u
\end{aligned}
$$

where

$$
\alpha=a / b
$$

We consider the following conditions:

$$
\begin{gathered}
x(0)=\text { specified }, \quad y(0)=0 \\
x\left(t_{f}\right)=\text { specified, } \quad y\left(t_{f}\right)=\text { free } \\
|u| \leqslant 1
\end{gathered}
$$

and

$$
\alpha<0, \quad c>0
$$

Initially the induced dipole is zero, so we have $y(0)=0$. Since we are only interested in the position of the particle and not interested in the final state of the induced dipole, we have $y\left(t_{f}\right)=$ free. Because the available voltage has a magnitude limit, we require $|u| \leqslant 1$. The condition $c>0$ comes from (4), but the condition $\alpha<0$ is arbitrary. The case of $\alpha>0$ can be handled similarly. When $\alpha=0$, then the system becomes an affine system, which is relatively easy to deal with. Because $b \neq 0$ generically in (3), the coefficient $\alpha$ in (20) is generically welldefined. Notice in (17) that depending on the sign of $b$ the original region $\left\{x_{2} \geqslant 0\right\}$ is mapped to

$$
\{x \geqslant 0\} \text { or }\{x \leqslant 0\}
$$

This gives a state constraint to the dynamics in (18) and (19). Equations (18) and (19) with (21)-(24) and a state constraint (25) are our final dynamics.

\subsection{Statement of the time-optimal problem}

We address the following time-optimal control problem:

Consider the system (18) and (19) with conditions (21)-(24). Find a time-optimal trajectory with the state constraint $x \geqslant 0$ (or $x \leqslant 0$ ).

The same time-optimal control problem without the state constraint was fully and analytically studied in Reference [14], summarized as follows. First, when $x\left(t_{f}\right)<x(0)$, there are no timeoptimal trajectories even though all $x\left(t_{f}\right)(<x(0))$ are reachable. Second, for $x\left(t_{f}\right)>x(0)$, timeoptimal trajectories exist if and only if the parameters satisfy $(1+\alpha c)>0$. Moreover, when $(1+2 \alpha c)>0$, the existence and uniqueness of time-optimal trajectories were proved, and the 
formula of optimal control was constructed. However, in the case of $(1+2 \alpha c) \leqslant 0$, only existence was shown. Instead of uniqueness, a finite algorithm for finding optimal trajectories was provided. Irrespective of the sign of $(1+2 \alpha c)$, a feature of all time-optimal trajectories is that there is an initial undershoot in $x(t)$. One can guess this from (18), (19) and $y(0)=0$ in (2I). Because of this initial undershoot, when the initial position $x(0)$ of $x$ is close to $x=0$, the timeoptimal trajectory without the state constraint violates the state constraint $x \geqslant 0$. This phenomenon leads us to study the time-optimal control of the same system with the state constraint.

\subsection{Numerical algorithm to construct optimal trajectories}

We make a numerical study of the time-optimal control problem given in Section 4.2. For convenience, we will only consider the case of the state constraint $x \geqslant 0$.

Let us introduce a time-scaling

$$
s=t / T
$$

for some $T>0$. Let $\left(z_{1}(s), z_{2}(s)\right)=(x(s T), y(s T))$. We use' to denote the derivative with respect to $s$. Let us first reformulate the time-optimal problem such that the control variable $u$ disappears and the time interval is normalized to $[0,1]$. The new idea of removing the control variable was effectively employed in the software package called nonlinear trajectory generation (NTG) to solve optimal control problems; see References [23,24]. Along these lines, the timeoptimal control problem is given by

$$
\left\{\begin{aligned}
\min _{\left(z_{1}, z_{2}\right) \in \mathbb{R}^{2}} T \\
\text { subject to } \\
\\
F\left(z_{1}, z_{2}, z_{1}^{\prime} / T, z_{2}^{\prime} / T\right)=0 \\
\left|z_{2}^{\prime} / T+c z_{2}\right| \leqslant 1 \\
z_{1} \geqslant 0 \\
z_{1}(0), z_{1}(1)=\text { specified } \\
z_{2}(0)=0 \\
T \geqslant \varepsilon
\end{aligned}\right.
$$

for a small $\varepsilon>0$ where

$$
F(x, y, \dot{x}, \dot{y})=\dot{x}-y(\dot{y}+c y)-\alpha(\dot{y}+c y)^{2}
$$

In (26), $\varepsilon$ can be chosen to be any sufficiently small positive number that ensures $T$ is positive. Notice that $(x(t), y(t), \dot{x}(t), \dot{y}(t))$ is replaced by $\left(z_{1}(s), z_{2}(s), z_{1}{ }^{\prime}(s) / T, z_{2}{ }^{\prime}(s) / T\right)$, which normalizes the time interval to $[0,1]$. The constraint $F=0$ in (26) comes from the substitution of $u=\dot{y}+c y$ in (19) to (18).

We now approximate this (continuous-time) optimal control problem by a (discrete) nonlinear dynamic programming. First, we represent $\left(z_{1}, z_{2}\right)$ with $B$-splines as follows:

$$
z_{1}(s ; q)=\sum_{i=1}^{p_{1}} B_{i, k_{1}}(s) C_{i}^{1}, \quad z_{2}(s ; q)=\sum_{i=1}^{p_{2}} B_{i, k_{2}}(s) C_{i}^{2}
$$


with

$$
\begin{aligned}
p_{j} & =l_{j}\left(k_{j}-m_{j}\right)+m_{j} \\
q & =\left(C_{1}^{1}, \ldots, C_{p_{1}}^{1}, C_{1}^{2}, \ldots, C_{p_{2}}^{2}\right) \in \mathbb{R}^{p_{1}+p_{2}}
\end{aligned}
$$

where $\left\{B_{i, k_{j}}(t), i=1,2\right\}$ is the $B$-spline basis function defined in Reference [25] for $z_{i}$ with order $k_{j}, C_{j}^{i}$ are the coefficients of the $B$-spline, $l_{j}$ is the number of knot intervals, and $m_{j}$ is number of smoothness conditions at the knots. The curve $\left(z_{1}, z_{2}\right)$ is thus represented by the coefficient vector $q . B$-splines have the advantage that it is easy to enforce continuity across knot points and to compute their derivatives.

We then discretize the time interval $[0,1]$ into $(N-1)$ subintervals

$$
0=s_{1}<s_{2}<\cdots<s_{N}=1
$$

In general $N$ collocation points $\left\{s_{1}, \ldots, s_{N}\right\}$ are chosen uniformly over the time interval $[0,1]$ for convenience although optimal knots placements may also be considered. Both dynamics and constraints will be enforced at the collocation points. The problem in (26) can be approximated by the following nonlinear programming form: subject to

$$
\left\{\begin{array}{l}
\min _{q \in \mathbb{R}^{p_{1}}+p_{2}} T \\
\text { subject to } \\
\\
F\left(z_{1}(s ; q), z_{2}(s ; q), z_{1}{ }^{\prime}(s ; q) / T, z_{2}{ }^{\prime}(s ; q) / T\right)=0 \\
\left|z_{2}{ }^{\prime} / T+c z_{2}\right| \leqslant 1 \\
z_{1} \geqslant 0 \\
z_{1}(0 ; q), z_{1}(1 ; q)=\text { specified } \\
z_{2}(0 ; q)=0 \\
T \geqslant \varepsilon
\end{array}\right\} \text { for every } s \in\left\{s_{1}, \ldots, s_{N}\right\}
$$

The coefficients of the $B$-spline basis functions can be optimized with nonlinear programming. We note that the resultant control law is sub-optimal because we allow only polynomials for $\left(z_{1}, z_{2}\right)$ and $u(s ; q)=z_{2}^{\prime}(s ; q) / T+c z_{2}(s ; q)$. However, as any continuous function on a closed interval can be uniformly approximated by polynomials according to the Stone-Weierstrass theorem [26], we can find sub-optimal trajectories which are sufficiently close to optimal ones.

We make a remark on the non-flatness of the system in (18) and (19); see References [27, 28] for the theory of flatness. A system is flat if one can find a set of outputs (equal in number to the number of inputs) such that all states and inputs can be determined from these outputs without integration (thus, differentiation is allowed). Hence, if systems (18)-(19) were flat, we could reformulate (26) with only one function (a flat output) and represent it with $B$-splines in (30), which would reduce the numerical load in nonlinear programming optimization [24,29]. 
However, systems (18)-(19) is not flat. It can be checked by the ruled-manifold criterion which is given in the following:

Theorem 4.1 (Martin et al. [28], Rouchon [30])

Assume the system $\dot{z}=f(z, u)$ is flat. The projection on the $p$-space of the submanifold $p=f(z, u)$, where $z$ is considered as a parameter, is a ruled manifold for all $z$.

Eliminating $u$ from the dynamics $\dot{z}=f(z, u), z \in \mathbb{R}^{n}$ yields a set of equations $F(z, \dot{z})=0$ that defines a ruled manifold. In other words for all $(z, p) \in \mathbb{R}^{2 n}$ such that $F(z, p)=0$, there exists a direction $d \in \mathbb{R}^{n}, d \neq 0$ such that

$$
\forall \lambda \in \mathbb{R}, \quad F(z, p+\lambda d)=0
$$

One can check that there is no such direction for systems (18)-(19), and thus our system is not flat. This non-flatness of systems (18) (19) explains why we used both states $x$ and $y$ (or, $z_{1}$ and $z_{2}$ ) in (26) (or (30)).

\subsection{Simulations}

We now perform a simulation to illustrate the difference of the time-optimal problem with the state constraint $(x \geqslant 0)$ and without it.

Consider the specification:

$$
\alpha=-3 / 4, \quad c=1 ; \quad x(0)=0.1, \quad x\left(t_{f}\right)=1.1
$$

We choose this arbitrarily for the purpose of comparison between the time-optimal control with and without the state constraint. If one wants to use a set of real data, then one needs to recall that $x$ in (18) is the transformed variable in (17). Also, one might need to modify (30) (or (26)) with a time-rescaling, a change of control bound, etc for the purpose of numerics.

According to Reference [14], the minimum time cost, $T_{\text {w.o.s.c. without the state constraint is }}$

$$
T_{\text {w.o.s.c. }}=7.8117
$$

A plot of $(x(t), y(t), u(t))$ in this case is given in Figure 6(a). Notice that the trajectory $x(t)$ has such an indershoot that it violates the state constraint. This initial undershoot is due to the existence of the term $\alpha u^{2}(\leqslant 0)$ in (18) and the initial condition $y(0)=0$.

We then performed a numerical computation of a time-optimal control with the state constraint. We choose

$$
k_{i}=6, \quad m_{i}=4, \quad l_{i}=9, \quad N=80
$$

with $i=1,2$ for the $B$-splines parameters in (28) and (29). The computed time cost is

$$
T_{\mathrm{w} . \mathrm{s.c.}}=8.6482
$$

The corresponding plot of $(x(t), y(t), u(t))$ is given in Figure 6(b). Notice in this case that the trajectory respects the state constraint, $x \geqslant 0$. We remark that only the control $u(t)$ was computed with (30). Then, we ran the simulation of the dynamics with this $u(t)$, so $(x(t), y(t))$ in Figure 6(b) is not the curve directly from (30) respecting the dynamics only on the $N$ collocation points, but the real trajectory satisfying the dynamics for all $t$. The comparison between the two plots in Figure 6 shows the necessity of the state constraint in finding time-optimal trajectories. Let us now consider a traditional approach. We assume that the signs of $a$ and $b$ in (2) are given 

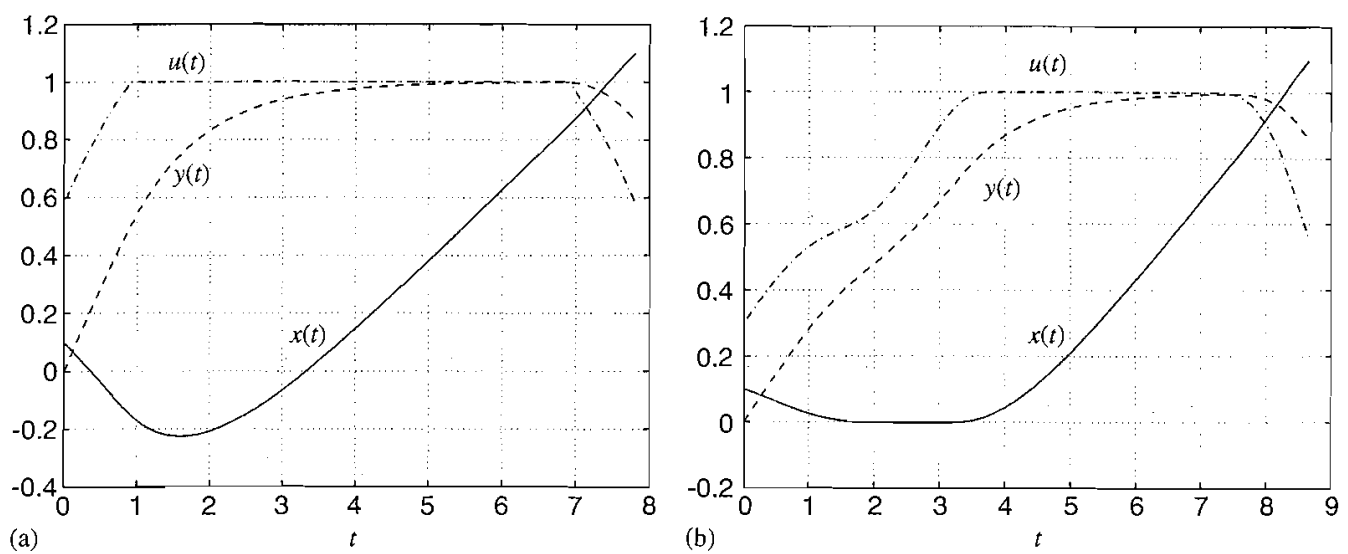

Figure 6. (a) A time-optimal trajectory $(x(t), y(t))$ corresponding to the optimal control $u(t)$ without the state constraint, $x \geqslant 0$; and (b) a time-optimal trajectory $(x(t), y(t))$ corresponding to the optimal control $u(t)$ with the state constraint, $x \geqslant 0$.

by $a<0$ and $b>0$ so that $\alpha=a / b=-0.75$ as given above. Then,

$$
\operatorname{Re}[G(\mathrm{j} \omega)]=a+\frac{b c}{\omega^{2}+c^{2}}=b\left(-0.75+\frac{1}{\omega^{2}+1}\right)
$$

As $\Delta x>0$, one would choose $\omega$ which maximizes $\operatorname{Re}[G(\mathrm{j} \omega)]$ because the real part of $G(\mathrm{j} \omega)$ in $(10)$ is a gain to the vertical DEP force as explained in Section 2.3. Thus, one would choose $\omega=0$. That is, $u=1$ or -1 . In either case, simple integration yields

$$
x(t)=0.25 t+\mathrm{e}^{-t}-0.9
$$

One can check that this trajectory violates the state constraint $x \geqslant 0$ as the lowest point along the trajectory is $x=-0.3034$ at $t=\ln 4$ and that the time cost $T_{\text {trad. }}$ to reach $x_{f}=1.1$ is

$$
T_{\text {trad. }}=7.9985
$$

Notice that in the traditional method it is not clear how to incorporate the state constraint into the control design procedure, but the state constraint is well treated by the time optimal control technique. We now compare the trajectory derived from the traditional method and the timeoptimal trajectory without the state constraint considered. From (31) and (33) we see that the time-optimal control improves the time cost by

$$
\frac{T_{\text {trad. }}-T_{\text {w.o.s.c. }}}{T_{\text {trad. }}} \times 100=2.335 \%
$$

It is interesting to notice that along the time-optimal trajectory without the state constraint considered one uses less energy $\left(\int|u|^{2}\right)$ and the magnitude of the undershoot is smaller than along the trajectory with $u=1$ or -1 . 


\section{CONCLUSIONS}

Since the initially significant study by Pohl [1], dielectrophoresis has been used for manipulating, separating and characterizing micro-/nano-/bio-particles. The objective of this paper is to invite control engineers to this application. After suggesting a list of future research directions for control engineers, we made a case study of the time-optimal control of a particle with dielectrophoresis. We derived the dynamics, and stated the time-optimal control problem with a state constraint, provided an NTG-approach nonlinear programming optimization algorithm to compute optimal trajectories and performed a simulation. The time-optimal control problem of the same system without the state constraint was already studied in Reference [14]. With the simulation, we compared the two cases: with or without the state constraint. The case study in Section 4 provides a good example of the synergy of engineering application and control theory. The former inspires the latter by providing new problems and the latter helps the former by providing solutions. We hope that this article stimulates control engineers so that they can enjoy the interdisciplinary research in nano/biotechnology through dielectrophoresis.

\section{ACKNOWLEDGEMENTS}

The first author would like to thank professor Igor Mezic for having introduced him to the topic of dielectrophoresis and having given him insight during his post-doctoral period at UCSB, and professor Noah Cowan at the Johns Hopkins University and professor Banavara Shashikanth at NMSU, Las Cruces for invaluable comments.

\section{REFERENCES}

1. Pohl HA. Dielectrophoresis. Cambridge University Press: Cambridge, 1978.

2. Greer NG, Morgan H. Dielectrophoretic separation of nano-particles. Journal of Physics D: Applied Physics 1997; 30(11):L41-L44.

3. Huang Y, Ewalt KL, Tirado M, Haigis TR, Forster A, Ackley D, Heller MJ, O'Connell JP. Electric manipulation of bioparticles and macromolecules on microfabricated electrodes. Analytical Chemistry 2001; 73:1549-1559.

4. Hughes MP. Strategies for dielectrophoretic separation in laboratory-on-a-chip systems. Electrophoresis 2002; 23(16):2569-2582.

5. Hughes MP. Nanoelectromechanics in Engineering and Biology. CRC Press: Boca Raton, 2002.

6. Video Library by the Dielectrophoresis Group in the Department of Molecular Pathology at the University of Texas, M.D. Anderson Cancer Center in Houston, Texas. http://www.dielectrophoresis.org/PagesMain/Video Library.htm [10 May 2005].

7. Zheng L, Brody JP, Burke PJ. Electronic manipulation of DNA, proteins, and nanoparticles for potential circuit assembly. Biosensors and Bioelectronics 2004; 20(3):606-619.

8. Jones TB. Electromechanics of Particles. Cambridge University Press: Cambridge, 1995.

9. Daniel VV. Dielectric Relaxation. Academic Press: New York, 1967.

10. Kaler KVIS, Jones TB. Dielectrophoretic spectra of single cells determined by feedback-controlled levitation. Biophysical Journal 1990; 57(1):173-182.

11. Kaler KVIS, Xie J-P, Jones TB, Paul R. Dual-frequency dielectrophoretic levitation of Canola protoplasts. Biophysical Joumal 1992; 63(1):58-69.

12. Chang DE, Loire S, Mezic I. Separation of bioparticles using the travelling wave dielectrophoresis with multiple frequencies. Proceedings of the IEEE Conference on Decision and Control, Hawaii, 2003.

13. Shapiro B. (ed.). Report from the NSF workshop on Control and Systems Integration of Micro- and Nano-scale Systems, 2004. http://www.isr.umd.edu/CMN-NSFwkshp/ [10 May 2005].

14. Chang DE, Petit N, Rouchon P. Time-optimal control of a particle in a dielectrophoretic system. IFAC Congress 2005, Prague, Czech Republic.

15. Jackson JD. Classical Electrodynamics (3rd edn). Wiley: New York, 1999. 
16. Cui L, Holmes D, Morgan H. The dielectrophoretic levitation and separation of latex beads in microchips. Electrophoresis 2001; 22:3893-3901.

17. Morgan H, Izquierdo AG, Bakewell D, Green NG, Ramos A. The dielectrophoretic and travelling wave forces generated by interdigitated electrode arrays: analytical solution using Fourier series. Jounnal of Physics $D$ : Applied Physics 2001; 34:1553-1561.

18. Chang DE, Loire S, Mezic I. Closed-form solutions in the electrical field analysis for dielectrophoretic and travelling wave interdigitated electrode arrays. Journal of Physics D: Applied. Physics 2003; 36(23):3073-3078.

19. Guckenheimer J, Holmes P. Nonlinear Oscillations, Dynanical Systems, and Bifurcations of Vector Fields. Springer: Berlin, 2000.

20. Purcell EM. Life at low Reynolds number. American Journal of Physics 1977; 45:3-11.

21. Ajdari A, Prost J. Mouvement induit par un potentiel périodique de basse symétrie: diélectrophorèse pulsée. Comptes Rendus de l'Académie Sciences, Paris 1992; 315(Série II):1635-1639.

22. Qian L, Scott M, Kaler KVIS, Pual R. Integrated planar concentric ring dielectrophoretic (DEP) levitator. Joumal of Electrostatics $2002 ; \mathbf{5 5 : 6 5 - 7 9 . ~}$

23. Milam MB. Real-time optimal trajectory generation for constrained dynamical systems. Ph.D. Thesis, California Institute of Technology.

24. Milam MB, Mushambi K, Murray RM. A new computational approach to real-time trajectory generation for constrained mechanical systems. Proceedings of the IEEE Conference on Decision and Control, Sydney, Australia, 2000.

25. de Boor C. A Practical Guide to Splines. Springer: Berlin, 1978.

26. Marsden JE, Hoffman MJ. Elementary Classical Analysis (2nd edn). W. H. Freeman: New York, 1993.

27. Fliess M, Lévine J, Martin P, Rouchon P. Flatness and defect of non-linear systems: introductory theory and examples. International Journal of Control 1995; 61(6):1327-1360.

28. Martin P, Murray RM, Rouchon P. Flat systems. Proceedings of the 4th European Control Conference, Plenary Lectures and Mini-Courses, Brussels, 1997; 211-264.

29. Petit N, Milam MB, Murray RM. Inversion based trajectory optimization. Proceedings of the IFAC Symposium on Nonlinear Control Systems Design (NOLCOS), 2001.

30. Rouchon P. Necessary condition and genericity of dynamics feedback linearization. Journal of Mathematical Systems and Estimation 1995; 5(3):345-358. 